\title{
Propiedades psicométricas de la Escala de Autoconcepto Académico (EAA) en estudiantes universitarios chilenos
}

\author{
Psychometric properties of the Academic Self-Concept Scale \\ in Chilean university students
}

\author{
Juan Méndez Vera ${ }^{\mathrm{a}, *}$, José Luis Gálvez Nieto ${ }^{\mathrm{a}}$ \\ ${ }^{a}$ Universidad de La Frontera, Chile
}

\section{Resumen}

El presente estudio tuvo como objetivo evaluar las propiedades psicométricas de la Escala de Autoconcepto Académico (EAA) en una muestra de estudiantes universitarios de Chile. Para ello, se realizó un estudio de corte transversal y se seleccionó a los participantes a través de un muestro probabilístico estratificado de 1290 estudiantes de ambos sexos (57\% mujeres y 43\% hombres), con una media de edad de 21.03 años. La estructura de la escala fue comprobada a través del análisis factorial exploratorio y confirmatorio, utilizando el procedimiento de muestras cruzadas. Los análisis realizados evidencian adecuadas propiedades psicométricas para su uso en estudiantes universitarios manteniendo una estructura de dos factores correlacionados que explican el $53.7 \%$ de varianza del constructo. Los valores alfa de Cronbach y omega de McDonald para el factor de Rendimiento Percibido fueron de $\alpha=.753$ y $\omega=.787$; y para el factor de Autoeficacia Académica de $\alpha=.802$ y $\omega=.867$. Los resultados permiten considerar que la EAA es un instrumento válido y confiable para evaluar el autoconcepto académico en estudiantes universitarios.

Palabras clave: validez, confiabilidad, análisis factorial, autoeficacia académica.

Para citar este artículo:

Méndez, J., \& Gálvez, J. (2018). Propiedades psicométricas de la Escala de Autoconcepto Académico (EAA) en estudiantes universitarios chilenos. Liberabit, 24(1), 131-145. doi: 10.24265/liberabit.2018.v24n1.09

\begin{abstract}
The current study aimed to evaluate the psychometric properties of the Academic Self-Concept Scale in a sample of university students from Chile. To that end, a cross-sectional study was conducted and participants were selected through a probabilistic hierarchical method, resulting in a sample size of 1290 students of both genders (57\% females and $43 \%$ males) with a mean age of 21.03 years. The structure of the scale was checked through an exploratory and confirmatory factor analysis using the cross-sample method. The analysis of the scale showed suitable psychometric properties to be used in university students, while maintaining its two-factor structure that explain $53.7 \%$ of the construct's variance. Cronbach's Alpha and McDonald's Omega regarding the Perceived Performance subscale were $\alpha=.753$ and $\omega=$ .787, and regarding the Academic Self-Efficacy subscale were $\alpha=.802$ and $\omega=.867$. The results enable us to conclude that the Academic Self-Concept Scale is a valid and reliable instrument to assess academic self-concept in university students.
\end{abstract}

Keywords: validity, reliability, factor analysis, academic self-efficacy.

Este es un artículo Open Access bajo la licencia Creative Commons Atribución-NoComercial-CompartirIgual 4.0

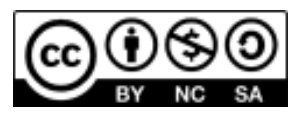




\section{Introducción}

\section{Contextualización: cambios en el acceso a la educación superior}

El panorama de la educación superior en América Latina ha cambiado considerablemente desde la segunda mitad del siglo xx y especialmente desde los años 90 en adelante, en donde se ha pasado de un modelo de educación superior elitista a uno en el que predomina la presencia de todos los sectores sociales en este nivel educativo. Esta demanda ha sido creciente en toda la región, al punto que hoy en día existen 14 millones de estudiantes universitarios en América Latina y el Caribe (United Nations Educational, Scientific and Cultural Organization [UNESCO], 1998).

De esta forma, de acuerdo con Chiroleu (2011), a la función clásica de las universidades de ser precursoras en la generación de conocimiento, se le suma la de generar espacios de movilidad social ascendente, especialmente en sociedades desiguales como las latinoamericanas. Por lo tanto, las universidades latinoamericanas tienen el desafío de transformarse y adaptarse para responder a la demanda de admisión y, junto con eso, deben preocuparse también por asegurar la permanencia y egreso de quienes cursan sus programas formativos.

Este escenario no es ajeno a la realidad chilena, donde la educación superior se encuentra en un proceso de cambio debido a distintas reformas y políticas que se están impulsando desde el Estado de Chile y que se han traducido en un alto incremento de la matrícula y una consecuente diversificación del perfil de ingreso, dando paso a nuevos grupos de estudiantes con diferentes características socioeconómicas y académicas (Brunner, 2015; Servicio de Información de Educación Superior [SIES], 2010; SIES, 2014). La masificación del sistema de educación superior chileno en los últimos años ha llegado casi a un $40 \%$ de cobertura neta en personas entre 18 y 24 años (SIES, 2014). Así, la educación superior, específicamente la universitaria, se encuentra altamente valorada por las familias chilenas en función del aumento de expectativas educacionales en el país (CIDE, como se citó en Canales, 2009), y también de los retornos económicos que implica un título profesional, el cual, según Psacharopoulos y Patrinos (2004), está cifrado sobre un $20 \%$ en relación a quiénes alcanzan solo la educación secundaria. De esta manera, la educación universitaria podría ser entendida como una herramienta para la movilidad social, sobre todo considerando que ya al 2003 cerca del 70\% de la matrícula del sistema en general correspondía a estudiantes que son primera generación de sus familias en ingresar a la universidad (Canales, 2009). Datos más recientes indican que en el año 2010 el 80\% de los estudiantes matriculados en la educación superior provenían de familias de las cuales eran primera generación (Meneses, Rolando, Valenzuela, \& Vega, 2010).

Sin embargo, pese a todos los esfuerzos que se han hecho para expandir las oportunidades de acceso a la educación superior, los desafíos en términos de inclusión y equidad son mayores (Espinoza \& González, como se citó en Bernasconi, 2015); ya que con el ingreso de jóvenes provenientes de niveles socioeconómicos más bajos al sistema educativo, se debe avanzar en promover oportunidades para progresar en sus estudios, graduarse y transitar exitosamente al mundo laboral. Ante este nuevo escenario, se torna relevante indagar en fenómenos como la masificación de la matrícula, la deserción, la retención, el rendimiento de los estudiantes universitarios (Garbanzo, 2007) y otras variables de tipo psicoafectivas que se encuentran asociadas al desempeño educativo, como el autoconcepto académico (Cazalla-Luna \& Molero, 2013).

\section{Autoconcepto académico: definición y asociaciones con variables psicosociales}

El autoconcepto académico se entiende como la parte del sí mismo que se relaciona más directamente con el rendimiento académico y que sirve como un 
sistema de guía personal para dirigir el comportamiento académico, jugando un rol fundamental en la determinación del rendimiento académico del sujeto (Villasmil, 2010). De esta forma, se trata de un constructo psicológico relativamente estable de percepciones sobre la evaluación de los propios comportamientos y atributos académicos (Schmidt, Messoulam, \& Molina, 2008). El autoconcepto académico es uno de los factores constituyentes del autoconcepto general (Shavelson, Hubner, \& Stanton, 1976), el cual se considera como una necesidad humana profunda y básica para tener una vida sana en términos generales y para lograr la autorrealización (Vera \& Zebadúa, como se citó en Cazalla-Luna \& Molero, 2013). Se relaciona con el bienestar general del individuo y se considera que muchos de los problemas psicológicos actuales, como la depresión o el fracaso académico, están relacionados o pueden tener un origen en un autoconcepto bajo (González \& Tourón, 1992). El autoconcepto no se hereda, sino que se desarrolla a partir de la acumulación de experiencias vividas por una persona a partir de su interacción con el ambiente (CazallaLuna \& Molero, 2013). Con el paso de los años, el autoconcepto se va estabilizando y comienza a determinar o dirigir la conducta de los individuos (García \& Musitu, 2014; González, 2005).

Investigaciones sobre el autoconcepto académico (Carranza \& Apaza, 2015; García \& Musitu, 2014; Herrera, Ramírez, \& Roa, 2004; Núñez, Martín-Albo, Navarro, \& Suárez, 2010) han demostrado una positiva relación entre todas las medidas de autopercepción y logro académico, confirmando que la medida del autoconcepto es el mejor pronosticador del logro académico, más aún que el coeficiente intelectual y la aptitud (Herrera et al., 2004).

Por el hecho de tratarse de una variable que podría explicar el éxito o el fracaso académico de los estudiantes, se han realizado diversos estudios que evalúan el autoconcepto académico en la educación formal principalmente en las etapas de educación básica y media (Amezcua \& Pichardo, 2000; Cerezo
\& Casanova, 2004; Fernandes, Marín, \& Urquijo, 2010; Gálvez-Nieto, Polanco, \& Salvo, 2017; Hilke \& Conway, como se citó en Padilla, García, \& Suárez, 2007; Pastor, Balaguer, \& Benavides, 2002). Los principales resultados hallados en estas dos etapas de la vida escolar indican que el autoconcepto académico es desarrollado en los primeros años de educación formal (Herrera et al., 2004) y que existen diferencias estadísticamente significativas entre niños y niñas en relación a la percepción que tienen de sí mismos en el ámbito académico, presentando los niños una mayor valoración en el autoconcepto (Pantoja \& Alcaide, 2013). Estas diferencias se mantienen durante los primeros años de educación formal ya que, a medida que se avanza hacia la adolecencia, las diferencias entre sexos se reducen hasta que ya no se identifican diferencias estadísticamente significativas entre hombres y mujeres (Amezcua \& Pichardo, 2000; Cerezo \& Casanova, 2004).

Ahora bien, en el ámbito de la educación superior los estudios que abordan la variable del autoconcepto académico son más escasos. Los resultados hallados en este nivel educacional indican que el autoconcepto académico es uno de los factores que permiten lograr un adecuado nivel de motivación académica en los estudiantes universitarios, ya que contribuye en la construcción de la confianza en sí mismo para enfrentar obstáculos cada vez más complejos (Carranza \& Apaza, 2015). Los hallazgos revelan que, en general, los estudiantes poseen un nivel de autoconcepto alto (Sánchez \& Matalinares, 2014), quizás por el solo hecho de ser estudiantes que han logrado ingresar a la educación superior.

En Chile, en el año 2012, Véliz y Apodaca evaluaron el autoconcepto académico, la autoeficacia académica y el bienestar psicológico en una muestra de 691 estudiantes universitarios chilenos. Sus resultados muestran que el autoconcepto académico es mejor en aquellos estudiantes que pertenecen a los últimos años de carrera por sobre los que ingresan a primer año. Además, evidencian la existencia de diferencias en el autoconcepto académico entre 
estudiantes pertenecientes a distintas disciplinas, donde los estudiantes pertenecientes a carreras relacionadas con el área de la salud tienen puntuaciones estadísticamente significativas más altas que los pertenecientes a otras disciplinas, como la educativa y psicosocial.

A nivel internacional, destaca la investigación desarrollada por Carranza y Apaza (2015), quienes se propusieron indagar la relación que existe entre autoconcepto académico y la motivación académica en una muestra de 92 estudiantes universitarios talentosos peruanos. Los resultados de la investigación confirman la existencia de una relación altamente significativa existente entre el autoconcepto académico y la motivación académica en los universitarios talentosos; esto quiere decir que ante un autoconcepto definido y equilibrado, se contribuye una estabilidad académica a nivel motivacional en los estudiantes universitarios.

Un nuevo estudio relacionado con la temática es el realizado por Sánchez y Matalinares (2014), quienes midieron el autoconcepto en una muestra de 217 estudiantes universitarios de Lima, Perú. Los hallazgos de la investigación revelan que los estudiantes que poseen un alto nivel de autoconcepto académico tienden a buscar en menor medida soporte y ayuda de parte de los pares, produciéndose una relación inversamente proporcional entre autoconcepto académico y búsqueda de soporte. De forma contraria, la relación se vuelve directamente proporcional al comparar el alto nivel de autoconcepto académico con los valores de conformidad y liderazgo. En este aspecto en particular, aquellos estudiantes que reportan un mayor autoconcepto académico tienden a estar más conformes consigo mismos y a desarrollar habilidades de liderazgo frente a sus pares, ya que al percibirse como buenos estudiantes sienten poseer, mayormente, la capacidad de ejercer el cargo de dirigir y tener autoridad sobre otras personas.
Otra investigación en ámbito universitario es la desarrollada por Pinilla, Montoya y Dussán (2013), quienes caracterizaron el autoconcepto en una muestra de 85 estudiantes universitarios de una universidad de Colombia. Los resultados del estudio arrojaron que, independientemente de la carrera que estudian, tanto hombres como mujeres del último año poseen un nivel de autoconcepto académico mayor que los de primer año, lo que demuestra que los estudiantes sienten más confianza en sí mismos en el ámbito académico a medida que avanzan en la carrera. De acuerdo con los autores, a medida que se acercan al término de su plan de estudios, los estudiantes validan sus sentimientos de autoeficacia académica frente a su desempeño en el contexto académico.

Como se ha presentado, el autoconcepto académico es un constructo de alto impacto en la vida académica de los estudiantes en todos sus niveles, considerando la importancia que poseen las autopercepciones y las creencias en relación a la eficacia para efectuar una conducta y alcanzar una meta, y las creencias respecto de sus posibles resultados (Bandura, 1987). Por tanto, la autoevaluación de los comportamientos y atributos personales respecto del desempeño académico, sería uno de los mejores predictores del logro académico (Román \& Musitu, 1982). Otro impacto relevante de destacar del autoconcepto académico en el ámbito educativo es que estaría relacionado con las decisiones que toman los estudiantes en cuanto a la persistencia y el esfuerzo con que enfrentan las actividades educativas, y el aprendizaje con el establecimiento de relaciones apropiadas con sus pares, con las expectativas académicas de su familia y con sus futuras aspiraciones (Schmidt et al., 2008). Por lo tanto, contar con un instrumento válido para la evaluación de este constructo permitiría obtener información sobre este, con lo cual se podría favorecer el desarrollo de un autoconcepto académico positivo y mejorar el desempeño y logros académicos de los estudiantes (Gálvez-Nieto et al., 2017), sobre todo en educación superior debido a la escasa investigación que existe en el área; considerando además, que la mejora de la calidad educativa es un desafío 
permanente para todas las instituciones de educación superior. Por ello, se vuelve esencial avanzar hacia el desarrollo de investigaciones basadas en indicadores de calidad relacionados con variables asociadas a las trayectorias educativas exitosas, por ejemplo, avanzando en el desarrollo de instrumentos y herramientas para predecir en que medida son capaces los estudiantes de alcanzar un buen rendimiento académico y terminar con éxito sus estudios (Corengia, Pita, Mesurado, \& Centeno, 2013).

\section{Estructura teórica y empírica de la Escala de Autoconcepto Académico (EAA)}

La medida del autoconcepto académico de las autoras Schmidt et al. (2008) es una escala que tiene como objetivo medir la autopercepción de la idea que el individuo tiene sobre su desempeño académico. Este instrumento es una medida útil para la evaluación psicoeducativa en el ámbito del desempeño académico $\mathrm{y}$, en términos prácticos, proporciona una medida precisa para la implementación de estrategias de apoyo académico y psicoeducativo.

El estudio psicométrico inicial planteado por Schmidt et al. (2008) y desarrollado en Argentina, propone una estructura de dos factores correlacionados denominados Rendimiento Percibido y Autoeficacia Académica. La escala original quedó compuesta por un total de 14 ítems, siete para cada factor, que en su totalidad explicaron un $43 \%$ de la varianza del constructo.

En esta línea, Gálvez-Nieto et al. (2017) se propusieron analizar los niveles de validez, fiabilidad e invarianza factorial de la EAA (Schmidt et al., 2008) en una muestra de estudiantes adolescentes chilenos. Los resultados de los análisis factoriales exploratorios y confirmatorios muestran que el constructo medido se mantiene estable, dando cuenta de la existencia de las dos dimensiones propuestas por la escala original; sin embargo, el factor Autoeficacia Académica sufrió la eliminación de uno de los ítems que no se ajustaba a la realidad de la muestra chilena. En tanto, los modelos de invarianza factorial aplicados a los grupos de hombres y mujeres participantes en el estudio señalan que la medida global de autoconcepto académico se mantiene equivalente entre ambos grupos.

Considerando los antecedentes expuestos y la escasez de instrumentos validados en población universitaria, la presente investigación se planteó la siguiente pregunta: ¿Cuáles son las propiedades psicométricas de fiabilidad y validez de la Escala de Autoconcepto Académico en una muestra de estudiantes universitarios chilenos? En base a la pregunta planteada, el objetivo general propuesto fue evaluar las propiedades psicométricas de la EAA en una muestra de estudiantes universitarios chilenos. A partir de los resultados de este estudio, se espera contar con un instrumento validado en población universitaria para Chile que permita generar información contextualizada sobre el autoconcepto académico y la generación de programas de apoyo académico y reducción de brechas en los estudiantes.

\section{Método}

\section{Participantes}

El muestreo realizado fue de tipo probabilístico estratificado (Pimienta, 2000). Los estratos corresponden a las seis facultades de una universidad de Temuco, Chile; y se buscó representatividad en relación al sexo y nivel socioeconómico, el cual fue calculado a partir de los quintiles socioeconómicos que corresponden a la medida oficial estandarizada para Chile para clasificar la población en relación a su nivel de ingresos y generar la entrega de apoyos socioeconómicos (Ministerio de Desarrollo Social, 2015). La muestra quedó constituida por 1290 casos, que representan el $14.68 \%$ de la población, de los cuales 737 correspondieron a mujeres (57\%) y 553 a hombres (43\%). En relación al nivel socioeconómico de los estudiantes, 324 casos pertenecen al quintil 1 (25.1\%); 279 casos, al quintil 2 (21.6\%); 232 casos, al quintil 3 (18.0\%); 207 casos, al quintil 4 (16.0\%) 
y 248 casos pertenecen al quintil 5 (19.2\%). Finalmente, la muestra por facultad quedó configurada por 375 casos (29.1\%) pertenecientes a la Facultad de Ingeniería y Ciencias; 323 casos (25.0\%), a la Facultad de Medicina; 293 casos (22.7\%), a la Facultad de Educación, Ciencias Sociales y Humanidades; 160 casos (12.4\%), a la Facultad de Ciencias Jurídicas y Empresariales; 76 casos (5.9\%), a la Facultad de Ciencias Agropecuarias y Forestales y 63 casos (4.9\%), a la Facultad de Odontología. La media de edad de los participantes fue de 21.03 años $(D . T .=2.62)$. Los hombres presentaron una media de edad de 21.16 años $(D . T$. $=2.85)$; las mujeres, una media de edad de 20.94 años $(D . T .=2.44)$.

\section{Instrumento}

La EAA, creada originalmente en Argentina por Schmidt et al. (2008), es una escala de autoreporte que evalúa el constructo del autoconcepto académico entendido como «la configuración organizada de percepciones relativamente estables que reflejan una evaluación de comportamientos y atributos académicos» (Schmidt et al., 2008, p. 103). La escala es autoadministrable y está dirigida a adolescentes escolarizados de 12 a 20 años.

La EAA se compone de 14 ítems a los que se responde mediante una escala tipo Likert de cinco puntos ( 1 = totalmente en desacuerdo, 5 = totalmente de acuerdo), los que se encuentran organizados en dos factores: Rendimiento Percibido, el cual se refiere a la percepción de rendimiento actual y posibles dificultades para el aprendizaje (ítems: 3, 7, 8, 9, 10, 11, 14) y Autoeficacia Académica, referido a la percepción de las propias capacidades académicas (ítems: 1, 2, 4, 5 , $6,12,13)$.

El comportamiento psicométrico, reportado por Schmidt et al. (2008), evidencia que la estructura factorial de la EAA explica un 43\% de la varianza total, donde el factor Rendimiento Percibido explica un 23.6\% y el factor Autoeficacia Académica, un 19.4\%. El análisis de confiabilidad, realizado a través del coeficiente alfa de Cronbach, mostró que los dos factores extraídos para los índices de consistencia interna (Rendimiento Percibido alfa de Cronbach = .68; Autoeficacia Académica alfa de Cronbach = .75).

Aplicaciones de la escala en una muestra de estudiantes secundarios chilenos evidencian adecuadas propiedades psicométricas, manteniendo la estructura de dos factores correlacionados que explican el $47.41 \%$ de la varianza del constructo. El análisis multigrupo reveló que la escala se mantiene invariante hasta el nivel de medias latentes $\left(\Delta \mathrm{SB}-\chi^{2}\right.$ $=28.061, p=.258 ; \Delta$ CFI $=-.004)$. La escala presentó adecuados índices de confiabilidad (factor Autoeficacia $\alpha=.739$ y factor Rendimiento $\alpha=.784$ ) (Gálvez-Nieto et al., 2017).

\section{Procedimiento}

La aplicación de la escala fue autoadministrada, donde la participación de los estudiantes fue voluntaria, pudiendo abandonar el estudio en el momento que considerasen oportuno. Los resguardos éticos fueron expresados a través de un consentimiento informado, el cual fue incluido en una sección previa a la escala, donde se explicó el objetivo general de la investigación y se esclarecieron de manera detallada asuntos éticos, tales como la voluntariedad, las consecuencias y los beneficios de la participación, y la utilización y el manejo de los datos recolectados. Previo a la aplicación del instrumento, se resolvieron dudas de los participantes relacionadas con el tiempo de duración y dudas puntuales con respecto al instrumento.

Como la Escala de Autoconcepto Académico (EAA) no presenta evidencias de validez en el contexto universitario chileno, los ítems se sometieron a revisión lingüística por tres jueces expertos que fueron seleccionados en base a dos criterios: conocimiento de la variable autoconcepto académico y cercanía con las terminologías propias de la cultura argentina. 


\section{Análisis de datos}

Con la base de datos depurada, se procedió a realizar los análisis estadísticos de los datos con diferentes software. En primer lugar, se dividió la muestra a través del procedimiento de Selección Aleatoria de Casos, que generó dos mitades aleatorias semejantes de 645 y 645 casos respectivamente.

Posteriormente, mediante factor (Lorenzo-Seva \& Ferrando, 2013), y con la primera mitad de la muestra ( $n=645$ ), se realiza el Análisis Factorial Exploratorio (AFE). Para la implementación del AFE, se utilizó una matriz policórica (Freiberg, Stover, De la Iglesia, \& Fernández, 2013). El método de extracción utilizado fue el de Mínimos Cuadrados no Ponderados (ULS) (Lorenzo-Seva, Timmerman, \& Kiers, 2011) y asumiendo que los factores presentarían correlaciones moderadas, una rotación Oblimin directo. Con la segunda mitad de la muestra $(n=645)$, se efectuó un Análisis Factorial Confirmatorio (AFC) con el software MPlus (Muthén \& Muthén, 2016), realizando análisis correspondientes al Índice de bondad de ajuste utilizando las pruebas del Promedio de los Residuales Estandarizados (RMSEA), el Índice de Ajuste Comparativo (CFI) y TLI (Moreno-Jiménez, MedaLara, Morante, Rodríguez, \& Palomera, 2006). Para los índices CFI y TLI, se considera un valor adecuado del modelo los valores mayores o iguales a .90 (Schumacher \& Lomax, 1996); mientras que para el RMSEA, se considera un ajuste razonable los valores inferiores a .08 (Browne \& Cudeck, 1993).

Finalmente, se evaluó la fiabilidad por consistencia interna del instrumento mediante el coeficiente alfa de Cronbach; sin embargo, considerando las limitaciones de este coeficiente (Trizano-Hermosilla \& Alvarado, 2016), se optó por complementar este análisis mediante el coeficiente omega de McDonald (McDonald, 1999), considerando valores aceptables los coeficientes mayores o iguales a .70 (Schmeiser \& Welch, 2006). Luego, y con el objetivo de estimar la homogeneidad de la escala, se utilizó el índice de correlación, ítem total corregido.

\section{Resultados}

\section{Validación por juicio de expertos}

La EAA de Schmidt et al. (2008) fue creada para ser aplicada originalmente a estudiantes adolescentes argentinos de educación secundaria; por lo que, en primer lugar, se procedió a realizar una adaptación de la escala mediante la técnica de validación por juicio de expertos con el propósito de adaptar el lenguaje y la terminología utilizados en las sentencias de la versión original a población universitaria chilena. Este análisis fue desarrollado por un grupo de académicos de la Universidad de La Frontera, obteniendo como resultado una adaptación del instrumento para el contexto universitario chileno, cuyos resultados empíricos indicaron que se debía incluir en los ítems 1 , 3 y 9 el concepto de «trabajos»; en los ítems 2 y 8 , sustituir el concepto «materias» por «asignaturas»; en los ítems 4 y 6, sustituir el concepto «año» por el de «semestre»; en el ítem 5, sustituir el concepto «escuela» por el de «universidad» y en el ítem 13, sustituir el concepto «cosa» por el de «materia». El reactivo de la escala adaptada se muestra en la Tabla 1.

\section{Análisis factorial exploratorio}

Para el AFE se consideró una primera muestra de 645 participantes correspondientes a la primera mitad de la muestra total obtenida tras la aplicación de la técnica de la validez cruzada (Murillo \& González, 2000). La prueba de adecuación muestral KMO (.897) y la prueba de esfericidad de Barlett $\chi^{2}(g l=91)=$ 2495.0, $p<.001$; indican que la matriz de datos es apropiada para la realización del AFE. Para determinar el número de dimensiones de la escala, se utilizó como procedimiento de análisis el método de Hull (LorenzoSeva et al., 2011), cuyos resultados muestran la presencia de dos factores correlacionados que en total explican un 51.19\% de la varianza total del constructo, donde el primer factor explica el $40.70 \%$ y el segundo factor el $10.49 \%$. Posteriormente, se analizaron cargas factoriales de la matriz rotada, lo que determinó la 
Tabla 1

Escala Autoconcepto Académico adaptada

\begin{tabular}{|c|c|c|c|}
\hline Ítem & REN & Ítem & AEA \\
\hline 3 & $\begin{array}{l}\text { Aunque preste atención a las } \\
\text { explicaciones que me dan los } \\
\text { profesores, los trabajos y tareas }\end{array}$ & 1 & $\begin{array}{l}\text { Soy capaz de hacer bien los } \\
\text { trabajos y tareas, aunque sean } \\
\text { difíciles. }\end{array}$ \\
\hline 7 & $\begin{array}{l}\text { no me salen. } \\
\text { Soy lento para aprender. }\end{array}$ & 2 & Si me esforzara lo suficiente, \\
\hline 8 & $\begin{array}{l}\text { No soy capaz de sacarme } \\
\text { buenas notas en las asignaturas. }\end{array}$ & 4 & Creo que podré obtener buenas \\
\hline 9 & $\begin{array}{l}\text { Cometo muchos errores cuando } \\
\text { hago trabajos y tareas de la } \\
\text { universidad. }\end{array}$ & 5 & $\begin{array}{l}\text { Aunque me esfuerce, siempre } \\
\text { me va a ir mal en la universidad }\end{array}$ \\
\hline 10 & $\begin{array}{l}\text { Me olvido fácilmente de lo que } \\
\text { aprendo. }\end{array}$ & 6 & $\begin{array}{l}\text { Soy capaz de mantener un buen } \\
\text { rendimiento a lo largo de todo }\end{array}$ \\
\hline 11 & Me cuesta entender lo que leo. & & un semestre. \\
\hline \multirow[t]{2}{*}{14} & Me resulta difícil estudiar. & 12 & $\begin{array}{l}\text { Soy capaz de realizar buenos } \\
\text { trabajos en clase. }\end{array}$ \\
\hline & & 13 & $\begin{array}{l}\text { Si me dedico a fondo, puedo } \\
\text { estudiar cualquier materia. }\end{array}$ \\
\hline
\end{tabular}

Nota : REN = Factor de rendimiento; AEA = Factor de autoeficacia.

existencia de dos valores que no se relacionan con las cargas factoriales expresadas por las autoras de la escala original (Schmidt et al., 2008). Específicamente, el ítem 3 presenta cargas factoriales en ambos factores: .437 en Autoeficacia Académica y .312 para el factor de Rendimiento Percibido. El ítem 8 es el que presenta la carga factorial más alta (.398) en el factor de Autoeficacia Académica, lo que resulta contradictorio con la propuesta original de la escala. Dado lo anterior, se eliminaron ambos ítems y se realizó nuevamente un AFE.
Los resultados de la prueba KMO (.884) y el estadístico de Bartlett $\chi^{2}(g l=66)=2052.1, p<.001$; ratifican la factibilidad de realizar nuevamente el AFE. Los resultados evidencian nuevamente la existencia de dos factores que en su conjunto explican el 53.77\% de la varianza total del constructo, mejorando significativamente el porcentaje de explicación de la varianza, donde el primer factor explicaría el 41.54\%, mientras que el segundo factor explicaría el 12.23\% (ver Tabla 2). De esta forma, el primer factor de Rendimiento Percibido quedó conformado por los ítems 7, 9, 10, 11 y 14; mientras que el segundo factor de Autoeficacia Académica, por los ítems 1, 2, 4, 5 , 6, 12 y 13. 
Tabla 2

Medias, desviaciones típicas (DT). Análisis Factorial Exploratorio y Confirmatorio

\begin{tabular}{|c|c|c|c|c|c|c|}
\hline \multirow{2}{*}{ Ítems } & \multirow{2}{*}{ Media } & \multirow{2}{*}{$D T$} & \multicolumn{2}{|c|}{ AFE } & \multicolumn{2}{|c|}{$\mathrm{AFC}$} \\
\hline & & & $\mathrm{RP}$ & AA & $\mathrm{RP}$ & $\mathrm{AA}$ \\
\hline $\begin{array}{l}\text { 1.- Soy capaz de hacer bien los trabajos y tareas, } \\
\text { aunque sean difíciles. }\end{array}$ & 4.01 & .785 & .151 & .526 & & $.720 *$ \\
\hline $\begin{array}{l}\text { 2.- Si me esforzara lo suficiente, podría aprobar } \\
\text { las asignaturas de este semestre. }\end{array}$ & 4.68 & 630 & -.138 & .787 & & $.621 *$ \\
\hline $\begin{array}{l}\text { 3.- Creo que podré obtener buenas notas este } \\
\text { semestre. }\end{array}$ & 3.94 & .850 & .050 & .667 & & $.769 *$ \\
\hline $\begin{array}{l}\text { 4.- Aunque me esfuerce, siempre me va a ir mal } \\
\text { en la universidad. }\end{array}$ & 1.58 & .857 & .127 & .596 & & $.666^{*}$ \\
\hline $\begin{array}{l}\text { 5.- Soy capaz de mantener un buen rendimiento } \\
\text { a lo largo de todo un semestre. }\end{array}$ & 3.60 & .917 & .179 & .584 & & $.764 *$ \\
\hline 6.- Soy lento para aprender. & 2.51 & 1.132 & 649 & .005 & $.689 *$ & \\
\hline $\begin{array}{l}\text { 7.- Cometo muchos errores cuando hago } \\
\text { trabajos y tareas de la universidad. }\end{array}$ & 2.39 & .962 & .494 & .157 & $.610^{*}$ & \\
\hline 8.- Me olvido fácilmente de lo que aprendo. & 2.72 & 1.084 & .705 & -.025 & $.683^{*}$ & \\
\hline 9.- Me cuesta entender lo que leo. & 2.47 & 1.091 & .669 & .001 & $.670^{*}$ & \\
\hline $\begin{array}{l}\text { 10.- Soy capaz de realizar buenos trabajos en } \\
\text { clase. }\end{array}$ & 3.85 & .847 & .126 & .548 & & $.671 *$ \\
\hline $\begin{array}{l}\text { 11.- Si me dedico a fondo, puedo estudiar } \\
\text { cualquier materia. }\end{array}$ & 4.38 & .821 & -.065 & .731 & & $.642 *$ \\
\hline 12.- Me resulta difícil estudiar. & 2.88 & 1.158 & .633 & .044 & $.604 *$ & \\
\hline
\end{tabular}

Nota: $* p<.001 ; \mathrm{RP}=$ rendimiento percibido; $\mathrm{AA}=$ autoeficacia académica.

\section{Análisis Factorial Confirmatorio}

Una vez obtenida la estructura factorial exploratoria, se procedió a realizar un Análisis Factorial Confirmatorio (AFC) utilizando la segunda parte de la muestra total obtenida de 645 casos con el propósito de confirmar el modelo teórico sugerido por el AFE. Utilizando el modelo de dos factores correlacionados, los índices de bondad presentaron los siguientes valores: $S B-\chi^{2}(g l=53)=237.949, p<.001 ; C F I=$ .956; TLI $=.945 ;$ RMSEA $=.071($ IC90\% $=.062-$ .080). Estos valores indican, en general, que el modelo ajusta bien a los datos, confirmando la estructura propuesta por el AFE. En la Tabla 2, se observan las cargas factoriales en donde, para el factor Rendimiento
Percibido, las cargas fluctúan entre .621 hasta un máximo de .769. Por otro lado, para el segundo factor, Autoeficacia Académica, las cargas fluctúan entre .604 y .689. Adicionalmente, la covarianza entre ambos factores es de .668, lo que representa un alto índice de asociación entre las variables latentes del modelo factorial subyacentes a la estructura empírica planteada.

\section{Análisis de fiabilidad}

El análisis de fiabilidad medido a través del coeficiente de alfa de Cronbach y omega de McDonald (1999) presenta valores aceptables. El factor de Rendimiento Percibido obtuvo un valor de $\alpha=.753$ 
y $\omega=.787$ y el factor de Autoeficacia Académica, un valor de $\alpha=.802$ y $\omega=.867$. Los resultados arrojados permiten indicar que ambos factores de la escala poseen adecuados niveles de consistencia interna dado que la eliminación de cualquier ítem, según el procedimiento de alfa de Cronbach si se elimina un elemento, bajaría la consistencia interna de los factores de la escala (ver Tablas 3 y 4).

Tabla 3

Análisis de confiabilidad Factor Rendimiento Percibido (RP)

\begin{tabular}{lll}
\hline Factor Rendimiento Percibido (RP) & C.I.T. & $\alpha$ E.E. \\
\hline 7. Soy lento para aprender. & .524 & .707 \\
9. Cometo muchos errores cuando hago & .449 & .733 \\
$\quad$ trabajos y tareas de la universidad. & & .578 \\
10. Me olvido fácilmente de lo que aprendo. & .552 & .688 \\
11. Me cuesta entender lo que leo. & .496 & .719 \\
14. Me resulta difícil estudiar. &
\end{tabular}

Nota: $\alpha=$.753; C.I.T. = correlación ítem total; E.E. = error estándar.

Tabla 4

Análisis de confiabilidad Factor Autoeficacia Académica (AEA)

Factor Autoeficacia Académica (AEA)

C.I.T.

$\alpha$ E.E.

1. Soy capaz de hacer bien los trabajos y tareas, aunque sean difíciles.

2. Si me esforzara lo suficiente, podría aprobar las asignaturas de este semestre.

4. Creo que podré obtener buenas notas este semestre.

5. Aunque me esfuerce, siempre me va a ir mal en la universidad.

6. Soy capaz de mantener un buen rendimiento a lo largo de todo un semestre.

12. Soy capaz de realizar buenos trabajos en clase.

13. Si me dedico a fondo, puedo estudiar cualquier materia. 


\section{Discusión}

El objetivo de esta investigación fue evaluar las propiedades psicométricas de la Escala de Autoconcepto Académico en una muestra de estudiantes universitarios chilenos. Los resultados que se discuten a continuación permiten concluir que la EAA presenta evidencia de calidad psicométrica para ser utilizada en estudiantes universitarios chilenos.

Los resultados del análisis factorial exploratorio realizado implementado a la primera muestra, evidenció una estructura teórica de dos factores correlacionados. Dicha solución factorial viene a aportar evidencias de validez que mantienen la estructura propuesta inicialmente por Schmidt et al. (2008) y los resultados obtenidos por Gálvez-Nieto et al. (2017); sin embargo, hubo una reducción de dos ítems que no alteró la capacidad explicativa del constructo. El AFC ratificó la estructura obtenida inicialmente, demostrando ser la mejor estructura para los datos chilenos.

Las evidencias de fiabilidad de la EAA demuestran que los ítems poseen una consistencia interna adecuada. Por su parte, el análisis de correlación ítem total, demostró adecuados niveles en cuanto al aporte de cada sentencia a la medición del constructo de autoconcepto académico. Además, el valor del coeficiente Omega para los dos factores fue adecuado con valores mayores o iguales a .787. Todos estos datos hacen concluir que la EAA entrega distintas evidencias de calidad psicométrica para su utilización en población universitaria chilena.

Entre las limitaciones del presente estudio, es posible señalar que la muestra utilizada, a pesar de ser representativa y amplia en términos numéricos, pertenece solo a una universidad chilena, aspecto que no garantiza la representatividad de las universidades chilenas. Futuros estudios podrían superar esta limitación incluyendo un mayor número de casas de estudio.
Se considera que esta investigación es pertinente y entrega aportes en la medida que permitió determinar el comportamiento psicométrico de la EAA en población universitaria chilena, lo que se considera relevante debido a que es factible contar con una escala que permite caracterizar el autoconcepto académico en estudiantes universitarios. Lo anterior se considera importante en la medida de que como país se han logrado avances significativos en el rubro de la educación superior, ya que el 59\% de los estudiantes que egresa de educación media ingresa a esta instancia (SIES, 2014). Sin embargo, y tal como lo señala el informe de la Organización para la Cooperación y el Desarrollo Económicos (OCDE, 2009), hoy en día es necesario una segunda generación de reformas que asuman el desafío de la calidad de la educación, ya que el reto no es solo que los estudiantes ingresen, sino que en este proceso exista inclusión y equidad para aquellos jóvenes más vulnerables. Además, el sistema internamente debe proveer a todos por igual las mismas oportunidades para avanzar en sus estudios, titularse e insertarse exitosamente en el mundo laboral.

Adicionalmente, es pertinente señalar que el presente trabajo constituye un primer aporte para evaluar el constructo del autoconcepto académico en universitarios de Chile, lo que se considera una contribución genuina para que otros estudios puedan ser llevados a cabo con el recurso de un instrumento con comprobadas propiedades psicométricas. Así también, la escala desarrollada puede aportar información significativa para realizar diagnósticos y llevar a cabo intervenciones en el plano académico universitario.

La validación de la EAA con una muestra de estudiantes universitarios chilenos evidencia la necesidad de llevar a cabo futuros estudios de validación en otros contextos universitarios latinoamericanos. Lo anterior se concluye debido a que el desafío en educación superior es permanente y amplio en Latinoamérica. Particularmente, en Chile se cuenta con una nueva configuración de estudiantes 
que ingresa a la educación superior con ciertas características que impactan positiva y negativamente en su trayectoria académica. Por ello, es preciso que las instituciones de educación superior asuman el desafío de generar conocimiento sobre este nuevo grupo, precisamente a través de investigaciones que permitan indagar en aquellas variables y elementos que influyen y repercuten en dichas trayectorias como se considera es el autoconcepto académico como conjunto de creencias y percepciones que van a guiar el comportamiento de los estudiantes en el ámbito de la educación formal.

\section{Conflicto de intereses}

Los autores declaran no contar con ningún tipo de conflicto de interés para la revisión y evaluación del manuscrito.

\section{Responsabilidad ética}

Los autores declaran que en la realización de este estudio no se han realizado experimentos en seres humanos ni en animales.

\section{Confidencialidad de los datos y consentimiento informado}

Los autores declaran que la participación en el estudio fue voluntaria y anónima, y se consideraron los resguardos éticos a través de la firma de un consentimiento informado previo a la aplicación del instrumento. Además, se declara que no se incorporan datos de los participantes que permitan que estos sean identificados.

\section{Referencias}

Amezcua, J., \& Pichardo, C. (2000). Diferencias de género en autoconcepto en sujetos adolescentes. Anales de Psicología, 16(2), 207-214.

Bandura, A. (1987). Pensamiento y acción. Buenos Aires: Paidós.

Bernasconi, A. (2015). La educación superior de Chile. Transformación, desarrollo y crisis. Santiago: Ediciones Universidad Católica de Chile.

Browne, M., \& Cudeck, R. (1993). Alternative ways of assessing model fit. In K. Bollen \& J. Long (Eds.), Testing structural equation models. Beverly Hills, CA: Sage.

Brunner, J. (2015). Un análisis de política de la política educacional. Debate Universitario, 3(6), 7-15.

Canales, A. (2009). Retención de estudiantes vulnerables en el sistema universitario. Revista Calidad de la Educación, 1(30), 50-83.

Carranza, R., \& Apaza, E. (2015). Autoconcepto académico y motivación académica en jóvenes talento de una universidad privada de Tarapoto. Revista Propósitos y Representaciones, 3(1), 233-263.

Cazalla-Luna, N., \& Molero, D. (2013). Revisión teórica sobre el autoconcepto y su importancia en la adolescencia. Revista Electrónica de Investigación y Docencia (REID), 1(10), 43-64.

Chiroleu, A. (2011). La educación superior en América Latina: ¿Problemas insolubles o recetas inadecuadas? Avaliação, 16(3), 631-653.

Cerezo, M. T., \& Casanova, P. (2004). Diferencias de género en la motivación académica de los alumnos de educación secundaria obligatoria. Revista Electrónica de Investigación Socioeducativa, 2(1), 97-112.

Corengia, A., Pita, M., Mesurado, B., \& Centeno, A. (2013). La predicción de rendimiento académico y deserción en estudiantes universitarios. Liberabit, 19(1), 101-112. Recuperado de http://revistaliberabit. com/por/revistas/liberabit19_1/9_corengia.pdf 
Fernandes, F., Marín, F., \& Urquijo, S. (2010). Relación entre los constructos autocontrol y autoconcepto en niños y jóvenes. Liberabit, 16(2), 217-226. Recuperado de http://revistaliberabit.com/es/revistas/ RLE_16_2_relacion-entre-los-constructosautocontrol-y-autoconcepto-en-ninos-y-jovenes.pdf

Freiberg, A., Stover, J., De la Iglesia, G., \& Fernández, M. (2013). Correlaciones policóricas y tetracóricas en estudios factoriales exploratorios y confirmatorios. Revista de Ciencias Psicológicas, 7(2), 151-164.

Gálvez-Nieto, J., Polanco, K., \& Salvo, S. (2017). Propiedades psicométricas de la Escala de Autoconcepto Académico (EAA) en estudiantes chilenos. Revista Iberoamericana de Diagnóstico e Avaliação Psicológica, 43(1), 5-16.

Garbanzo, G. (2007). Factores asociados al rendimiento académico en estudiantes universitarios, una reflexión desde la calidad de la educación superior pública. Educación Universidad de Costa Rica, 31(1), 43-63.

García, F., \& Musitu, G. (2014). AF5 Autoconcepto Forma 5. Madrid: TEA Ediciones.

González, M., \& Tourón, J. (1992). Autoconcepto y rendimiento escolar. Sus implicaciones en la motivación y en la autorregulación del aprendizaje. Pamplona: Navegraf.

González, Ó. (2005). Estructura multidimensional del autoconcepto físico. Revista de Psicodidáctica, 10(1), 121-130.

Herrera, F., Ramírez, M., \& Roa, J. (2004). ¿Cómo interactúan el autoconcepto y el rendimiento académico, en un contexto educativo pluricultural? Revista Iberoamericana de Educación, 2(37) 1-9.

Lorenzo-Seva, U., \& Ferrando, P. (2013). FACTOR 9.2 A Comprehensive program for fitting exploratory and semiconfirmatory factor analysis and IRT models. Applied Psychological Measurement, 37(6), 497498. doi: 10.1177/0146621613487794

Lorenzo-Seva, U., Timmerman, M., \& Kiers, H. (2011). The hull method for selecting the number of common factors. Multivariate Behavioral Research, 2(46), 340-364. doi: 10.1080/00273171.2011.564527
McDonald, R. (1999). Test theory: a unified treatment. Mahwah, NJ: Lawrence Erlbaum Associates.

Meneses, F., Rolando, R., Valenzuela, M., \& Vega, M. (2010) Ingreso a la educación superior: la experiencia de la cohorte de egreso 2005. Chile: Sistema Nacional de Información de la Educación Superior (SIES) y División de Educación Superior del MINEDUC.

Ministerio de Desarrollo Social (2015). Encuesta de caracterización socioeconómica nacional - CASEN. Santiago, Chile. Recuperado de http://www.ministerio desarrollosocial.gob.cl/pdf/upload/IDS2.pdf

Moreno-Jiménez, B., Meda-Lara, R., Morante, M., Rodríguez, A., \& Palomera, A. (2006). Validez factorial del Inventario de Burnout de psicólogos en una muestra de psicólogos mexicanos. Revista Latinoamericana de Psicología, 38(3), 445-456.

Murillo, C., \& González, B. (2000). Validación del modelo de regresión: contrastes de especificación incorrecta y contrastes de especificación. En C. Murillo, \& B. González. Manual de Econometría (pp. 1-35). Barcelona: Universidad Pompeu Fabra.

Muthén, L., \& Muthén, B. (21 de mayo de 2016). Mplus. Recuperado de https://www.statmodel.com/index. shtml

Núñez, J., Martín-Albo, J., Navarro, J., \& Suárez, Z. (2010). Adaptación y validación de la versión española de la Escala de Motivación Educativa en estudiantes de educación secundaria postobligatoria. Revista Estudios de Psicología, 31(1), 89-100.

Organización para la Cooperación y el Desarrollo Económicos (2009). Revisión de políticas nacionales de educación en Chile. París: OCDE.

Padilla, M., García, S., \& Suárez, M. (2007). Diferencias de género en el autoconcepto general y académico de estudiantes de $4^{\circ}$ de ESO. Revista de Educación, 1(352), 495-515.

Pantoja, A., \& Alcaide, M. (2013). La variable género y su relación con el autoconcepto y el rendimiento académico del alumnado universitario. Revista científica electrónica de Educación y Comunicación en la Sociedad del Conocimiento, 1(13), 124-140. 
Pastor, Y., Balaguer, I., \& Benavides, G. (2002). Influencia de los dominios del autoconcepto en la autoestima de los adolescentes. Revista de psicología social aplicada, 12(3), 97-112.

Pimienta, R. (2000). Encuestas probabilísticas vs. no probabilísticas. Política y Cultura, 1(13), 263-276.

Pinilla, V., Montoya, D., \& Dussán, C. (2013). Caracterización del autoconcepto en una muestra de estudiantes universitarios de los programas de ciencias sociales e historia de la ciudad de Manizales. Revista Latinoamericana de Estudios Familiares, 5(1), 106-123.

Psacharopoulos, G., \& Patrinos, H. (2004). Returns to investment in education: a further update. Education Economics, 12(2), 111-134.

Román, J. M., \& Musitu, G. (1982) Autoconcepto: una revisión de estudios empíricos. Universitas Tarraconensis. Revista de Psicología, Pedagogía y Filosofía, 4(2), 205-220.

Sánchez, R., \& Matalinares, M. (2014). Valores interpersonales y autoconcepto en estudiantes universitarios de la carrera de Ingeniería Ambiental y Administración de Empresas de una universidad estatal. Revista de Investigación en Psicología, 17(1), 55-80.

Schmeiser, C., \& Welch, C. (2006). Test development. En R. Brennan (Ed.). Educational Measurement (pp. 307-353). Westport: Praeger Publishers.

Schmidt, V., Messoulam, N., \& Molina, F. (2008). Autoconcepto académico en adolescentes de escuelas medias: presentación de un instrumento para su evaluación. Revista Iberoamericana de Diagnóstico e Avaliação Psicológica, 1(25), 81-106.

Schumacher, R., \& Lomax, R. (1996). A beginner's guide to structural equation modeling. New Jersey: Lawrence Erlbaum Associates, Publishers.
Shavelson, R., Hubner, J. S., \& Stanton, G. (1976). SelfConcept: validation of construct interpretations. Review of Educational Research, 46(3), 407-441.

Servicio de Información de Educación Superior (2010). Panorama de la educación superior en Chile. División de Educación Superior. Santiago: Ministerio de Educación.

Servicio de Información de Educación Superior (2014). Panorama de la educación superior en Chile 2014. Ministerio de Educación, Chile, Servicio de Información de Educación Superior. Santiago: Ministerio de Educación.

Trizano-Hermosilla, I., \& Alvarado, J. (2016). Best alternatives to Cronbach's Alpha reliability in realistic conditions: congeneric and asymmetrical measurements. Frontiers in Psychology, 7, 1-8. doi: 10.3389/fpsyg.2016.00769

United Nations Educational, Scientific and Cultural Organization (1998). Conferencia mundial sobre la educación superior. La educación superior en el siglo XXI visión y acción. Informe final. París: UNESCO.

Véliz, Á., \& Apodaca, P. (2012). Niveles de autoconcepto, autoeficacia académica y bienestar psicológico en estudiantes universitarios de la ciudad de Temuco. Revista Salud y Sociedad, 3(2), 131150.

Villasmil, J. (2010). El autoconcepto académico en estudiantes universitarios resilientes de alto rendimiento: un estudio de caso (tesis doctoral). Universidad de Los Andes, Venezuela. 


\section{Juan Méndez Vera}

Universidad de La Frontera (Chile)

Magíster en Educación con mención en Evaluación Educacional, su línea de investigación está vinculada a la educación superior, focalizándose en factores que intervienen en las trayectorias educativas de los estudiantes.

ORCID: 0000-0002-1485-925X

Autor corresponsal: juan.mendez@ufrontera.cl

\section{José Luis Gálvez Nieto}

Universidad de La Frontera (Chile)

Dr. en Ciencias de la Educación, cuenta con diversas publicaciones en el ámbito psicosocial. Su línea de investigación es clima escolar, actitud hacia la autoridad institucional, violencia escolar y resiliencia académica.

ORCID: 0000-0001-7642-8204

jose.galvez@ufrontera.cl 